
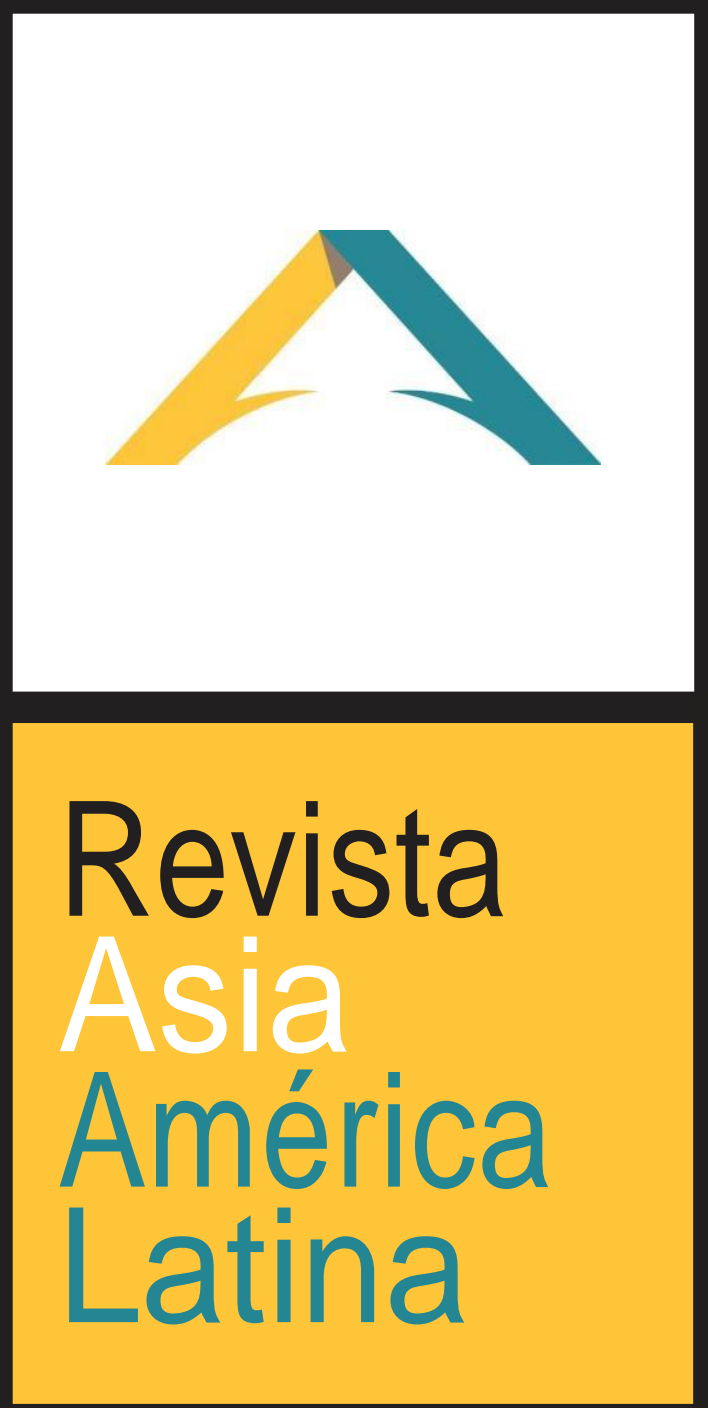

ISSN 2524-9347

Grupo de Estudios sobre Asia y América Latina Instituto de Estudios de América Latina y el Caribe Universidad de Buenos Aires

EU

DE BA 


\section{REPENSAR LA GOBERNANZA FINANCIERA GLOBAL DESDE} LA ASEAN

Lola Ibáñez

lolaibanez16@gmail.com

Universidad Nacional de Rosario

\section{Introducción}

El debate sobre la configuración de una gobernanza financiera global cobró renovado vigor luego de la profunda crisis de 2008. Una vez más, la cuestión de la regulación financiera recuperó relevancia en medio de una grave debacle económica, de la cual prácticamente ningún país quedó exento. El mercado hipotecario estadounidense se transformó en el epicentro de esta crisis, que se trasladó principalmente a los demás países desarrollados, pero también alcanzó al mundo en desarrollo bajo la forma de una pronunciada caída en la actividad económica (comercio, inversiones y flujos de capital).

Más precisamente, su origen tuvo lugar en un sector minoritario en el mercado de las hipotecas, como lo fueron las hipotecas subprime o de alto riesgo, que representaban poco más del $20 \%$ del total de hipotecas generadas. ${ }^{1}$ Cuando la tasa de impago de estas hipotecas sobrepasó el 30\%, ${ }^{2}$ grandes bancos de importancia sistémica, como Lehman Brothers, e instituciones respaldadas por el gobierno, como Fannie Mae y Freddie Mac, se declararon en bancarrota. Aquellas instituciones que no quebraron reportaron grandes problemas de solvencia debido a los altos niveles de endeudamiento que manejaban.

Claramente, el hecho de que un segmento minoritario del mercado hipotecario diera origen a una crisis de tal magnitud indicó que los actores financieros se habían conducido tan irresponsablemente, que el sistema no resistía la más mínima perturbación. Esto puso nuevamente sobre la mesa la problemática de cómo controlar el accionar imprudente de las grandes instituciones financieras, que adoptaban prácticas cuestionables independientemente del grado de riesgo en el que incurrían, y devolvió el foco a la figura del Estado como actor necesario e irrenunciable en la regulación y prevención de nuevas crisis.

\footnotetext{
${ }^{1}$ https://www.wsj.com/articles/lenders-step-up-financing-to-subprime-borrowers1424296649

2https://www.richmondfed.org/ /media/richmondfedorg/banking/markets_trends_and _statistics/trends/pdf/delinquency_and_foreclosure_rates.pdf
} 
Las características del sistema financiero actual vuelven ineludible la tarea de construir un nuevo modelo de gobernanza global, capaz de poner un coto a esta clase de comportamientos desmesurados que amenazan la estabilidad económica global. Su complejidad, opacidad y aparente homogeneidad lo tornan un sistema robusto pero frágil e incierto, que se está volviendo más propenso a crisis y donde la liquidez es una preocupación cada vez mayor (León et al., 2011).

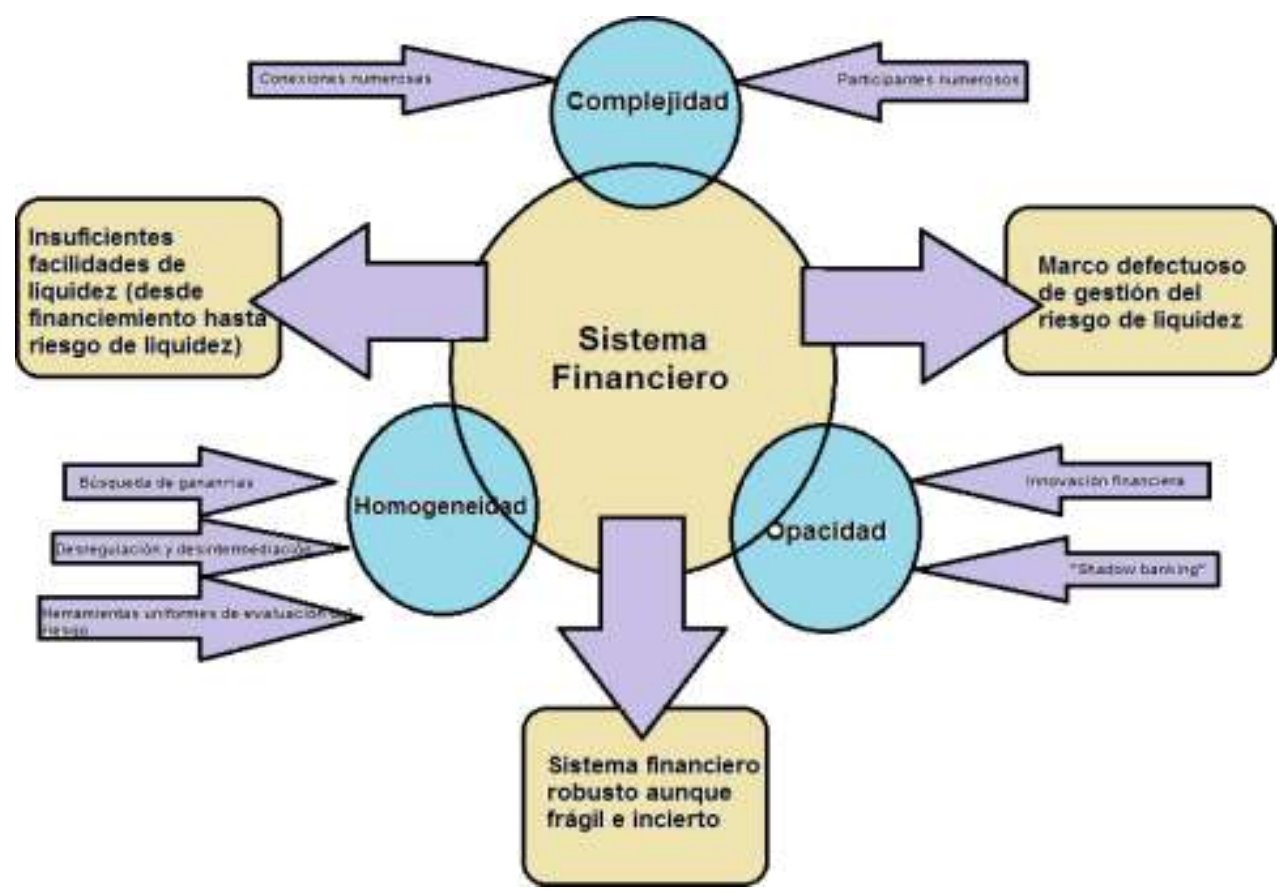

Gráfico 1: Esquema de sistema financiero propenso a crisis, problemas y desafíos. Fuente: León et al., 2011. Traducción propia.

De esta compleja interacción de características y debilidades, surge la noción de Too connected to fail (TCTF), similar al ya conocido Too big to fail, pero con énfasis en el fenómeno de la interconexión más que en el volumen de capital. Las instituciones too connected to fail se definen como aquellas cuyo nivel de conectividad, directa o indirecta, es tal que su incapacidad para cumplir con sus obligaciones resulta en la incapacidad de otros participantes o instituciones del sistema para cumplir con las suyas propias.

Con estas consideraciones en mente, es imperativo procurar construir un nuevo modelo global de gobernanza financiera que prevenga la repetición de crisis como la del 2008 y postule un paradigma regulatorio eficiente y 
efectivo. Para esto, es esencial que los países en desarrollo participen activamente y capitalicen de su presencia en los foros de los que forman parte. Los procesos de integración regional, por ejemplo, son una excelente herramienta para alcanzar acuerdos y concertar posiciones que luego puedan ser proyectadas y sostenidas a mayor escala.

Actualmente, presenciamos un período que se replantea seriamente los aspectos más controversiales de la integración global real, como la inmigración y los conflictos comerciales. Sin embargo, los actores internacionales parecen relegar una tarea no menos urgente y aún más desafiante, como lo es el abordaje de las repercusiones de la integración global financiera, en un contexto con un altísimo grado de interconexión y una complejidad que se eleva progresivamente. Si pensamos en la gobernanza global como aquel sistema generalizado que regula las cuestiones humanas a nivel mundial (O’Brien y Williams, 2004), podemos detectar la importancia que posee la formulación de las normas que regulan estas cuestiones. Importancia aún mayor si tenemos en cuenta que se refiere a mercados internacionales en los que se intercambian altísimos niveles de activos, y cuyo accionar en las últimas décadas se ha caracterizado por ser volátil y altamente reversible, viéndose al mismo tiempo afectado por comportamientos grupales y de contagio. Estas particularidades guardan en sí mecanismos de repetición de las crisis que ya hemos experimentado, y factores amplificadores de su gravedad.

De esta forma, es de suma importancia la participación de los países en desarrollo se aboque a la construcción de una nueva gobernanza financiera global, que les permita consolidar sus logros, proteger la estabilidad económica y promover un paradigma más equitativo. Se trata, en definitiva, de abogar por una verdadera alternativa al actual modelo de privatización de las ganancias y socialización del riesgo que termina siempre operando en detrimento del desarrollo.

En consonancia con esto, es indispensable que el peso de los países asiáticos en los organismos multilaterales se condiga de una vez con su lugar estratégico en la economía mundial. Richard Stubbs (2014) señala cómo las contribuciones de la Asociación de Naciones del Sudeste Asiático (ASEAN) a las cuestiones globales son generalmente vistas como marginales. Esto se debe, en gran parte, a la limitada influencia que posee sobre las mayores organizaciones mundiales, como ONU, el FMI, la Organización Mundial del Comercio y el Banco Mundial. Estas organizaciones ya estaban bien establecidas mucho antes de que la ASEAN pudiera comenzar a ejercer cualquier influencia, sin mencionar que sus prácticas y procedimientos son difíciles de cambiar. Sin embargo, prosigue el autor, por sí solo esto constituye una visión simplista que no reconoce la influencia internacional que ha alcanzado la Asociación, y la interesante alternativa paradigmática que ofrece ante el modelo occidental liberal de gobernanza global. 
En los siguientes apartados, relevaremos las particularidades económicas de la ASEAN como proceso de integración regional en el contexto global y el proceso de integración monetaria que tiene lugar al interior de esta región, para destacar las potencialidades de esta organización en la construcción de un nuevo paradigma de gobernanza financiera.

\section{La ASEAN a contracorriente de las tendencias actuales}

Como señalamos en la introducción, la crisis financiera de 2008 produjo cambios en los marcos regulatorios y compromiso por parte de actores políticos para promover la innovación institucional, aunque no así en el statu quo prevalente. El lugar central del que gozaban las economías del G20 y la Unión Europea en la gobernanza económica no fue alterado a raíz de la crisis; sin embargo, en la cumbre del G20 en Pittsburgh en septiembre de 2009 se operó un cambio en la orientación de las iniciativas de los organismos financieros internacionales.

La crisis pareció promover un espíritu organizacional tendiente, en mayor o menor medida, a operar proceso de pluralización de las instancias decisionales. En la Cumbre de Pittsburgh se propuso una revisión de cuotas del FMI y del Banco mundial, en menor medida, que reflejaran la importancia de los países en desarrollo (Kalher, 2010). De la misma forma, el Foro de Estabilidad Financiera (Financial Stability Forum o FSF) pasó a ser el Consejo de Estabilidad Financiera (Financial Stability Council o FSC), entidad sucesoria que sumó cinco países miembros. De estos países, tres no pertenecían al G20 (Singapur, Hong Kong y Suiza), uno pertenecía agregado dentro de la Unión Europea (Países Bajos) y el último pertenecía al G20, pero no al que otrora era el FSF (China)

Es interesante notar que la incorporación de economías asiáticas emergentes como Singapur y Hong Kong, además de la adhesión de China a un organismo de gobernanza como es el FSC, constituye un reconocimiento al traslado hacia continente asiático de una significativa porción del peso económico global. No obstante, la influencia de Asia en la gobernanza financiera global sigue sin ser consistente con su peso en la economía global (Henning y Khan, 2011). Asimismo, la presencia de países como India, China, Corea del Sur e Indonesia en el seno del G20 representa una ventana de oportunidad para influir en las altas esferas de decisión del escenario internacional.

Hoy en día, podemos afirmar que es pertinente hablar de un escenario económico internacional complejo y altamente volátil. La guerra comercial entre Estados Unidos y China ha venido a consolidar el pesimismo originado en las escasas perspectivas de crecimiento previstas para este 2019. Ya a fin del primer semestre de este año el Banco Mundial calificó al 2019 como un año 
con tensiones agudizadas e inversiones disminuidas, ${ }^{3}$ durante el cual se espera una alarmante caída del crecimiento económico mundial que lo pondría a nivel de un año conflictivo como fue 2016

Sin embargo, a más de cincuenta años de su creación, la ASEAN es uno de los procesos de integración regional de larga data que en la actualidad conserva un dinamismo económico y político activo, en comparación a otros procesos de integración que se encuentran atravesando diversas dificultades al interior de sus estructuras. Basta mirar hacia Europa para ver los desafíos que ha planteado el Brexit en términos de unidad y costos políticos, y las implicancias regionales y extrarregionales que conllevan el abandono de Gran Bretaña de su lugar en el foro europeo.

Del mismo modo, la realidad regional del Cono Sur se presenta se presenta compleja y plagada de desafíos para un proceso de integración que siempre ha estado altamente expuesto a los cambios de signo político de los gobiernos de la región, y que ahora debe lidiar además con miembros que experimentan importantes crisis económicas, rupturas del orden constitucional y una relación espinosa entre los dos mayores países integrantes.

De esta forma, la distintiva situación macroeconómica de la que gozan las economías del Sudeste Asiático no es solo una particularidad favorable, sino que, a la vez, que se configura en una plataforma desde la cual estos países pueden construir las bases de una política exterior más proactiva desde la cual poder proyectarse más asertivamente. En este caso, uno de los hechos más notables que se da en el marco de la ASEAN es el progresivo y sostenido crecimiento económico que han alcanzado los países miembros, situando el promedio regional en un elevado 5,1\%.

\begin{tabular}{|c|c|c|c|c|c|c|c|c|c|c|c|}
\hline II Etonomy & $\begin{array}{l}\text { Brand } \\
\text { Ounumilam }\end{array}$ & Cambodal & Fidsanein & LASPCR & Babapia & Myminar & Pillppine & Sngupsen & EThailund & Vist Man & ASEAX \\
\hline COP at curant pros (US50) & 13.6 & 245 & 1,0416 & 18.t & 354.2 & 71.5 & 342.6 & 3610 & 5051 & 289.7 & 29729 \\
\hline COP per capta (USS) & $31,685,1$ & 1,5355 & 3,5287 & 2,6278 & 10.907 .7 & 1.328 .2 & 3,2133 & 64.2739 & $7,433.5$ & 2.502 .6 & 4.577 .1 \\
\hline hea GOP prout (S) & 0.1 & 70 & 52 & 67 & 4.6 & 64 & 62 & 33 & 4.1 & 7.1 & 51 \\
\hline Ifatine ate anrago ofy & 0.2 & na & 32 & 20 & 0.9 & 69 & 53 & 04 & 1.1 & 35 & 0.2 .69 \\
\hline
\end{tabular}

Tabla 1: PBI por país integrante de la ASEAN.

Fuente: ASEAN Economic Integration Brief, 2019

Las economías emergentes asiáticas comenzaron su recuperación de la crisis financiera antes y más robustamente que sus contrapartes en otras regiones en desarrollo (Goldstein y Xie, 2009). Incluso podemos observar que

${ }^{3}$ https://www.bancomundial.org/es/publication/global-economic-prospects 
el dinámico desempeño económico de los países miembros de la ASEAN se presenta como una excepción que contrasta el crecimiento ralentizado generalizado entre sus pares occidentales y de algunas economías vecinas, como Japón.

\begin{tabular}{|l|r|r|r|r|r|r|r|}
\hline & 2015 & 2016 & 2017 & 2018 & 2019 & 2020 & 2021 \\
\hline Mundo & $\mathbf{2 , 9}$ & $\mathbf{2 , 6}$ & $\mathbf{3 , 1}$ & $\mathbf{3 , 0}$ & $\mathbf{2 , 6}$ & $\mathbf{2 , 7}$ & $\mathbf{2 , 8}$ \\
\hline Economías avanzadas & $\mathbf{2 , 3}$ & $\mathbf{1 , 7}$ & $\mathbf{2 , 3}$ & $\mathbf{2 , 1}$ & $\mathbf{1 , 7}$ & $\mathbf{1 , 5}$ & $\mathbf{1 , 5}$ \\
\hline Estados Unidos & 2,9 & 1,6 & 2,2 & 2,9 & 2,5 & 1,7 & 1,6 \\
\hline Zona euro & 2,1 & 2,0 & 2,4 & 1,8 & 1,2 & 1,4 & 1,3 \\
\hline Japón & 1,2 & 0,6 & 1,9 & 0,8 & 0,8 & 0,7 & 0,6 \\
\hline
\end{tabular}

Tabla 2: Perspectivas de crecimiento para 2019, 2020 y 2021.

Fuente: Banco Mundial

Vietnam es el país de la ASEAN con la mayor tasa de crecimiento y cuyo PBI ha experimentado una impresionante expansión en la última década, superando incluso las expectativas del Banco Mundial para 2018 que preveían un $6,8 \%{ }^{4}$ para ubicarse casi en el $7,1 \%$. Los sectores de la economía vietnamita que más dinamismo mostraron durante el primer cuarto del 2019 fueron la industria $(2,68 \%)$, los servicios $(6,5 \%)$, la construcción $(8,63 \%)$ y la manufactura y procesamiento $(12,35 \%) .^{5}$

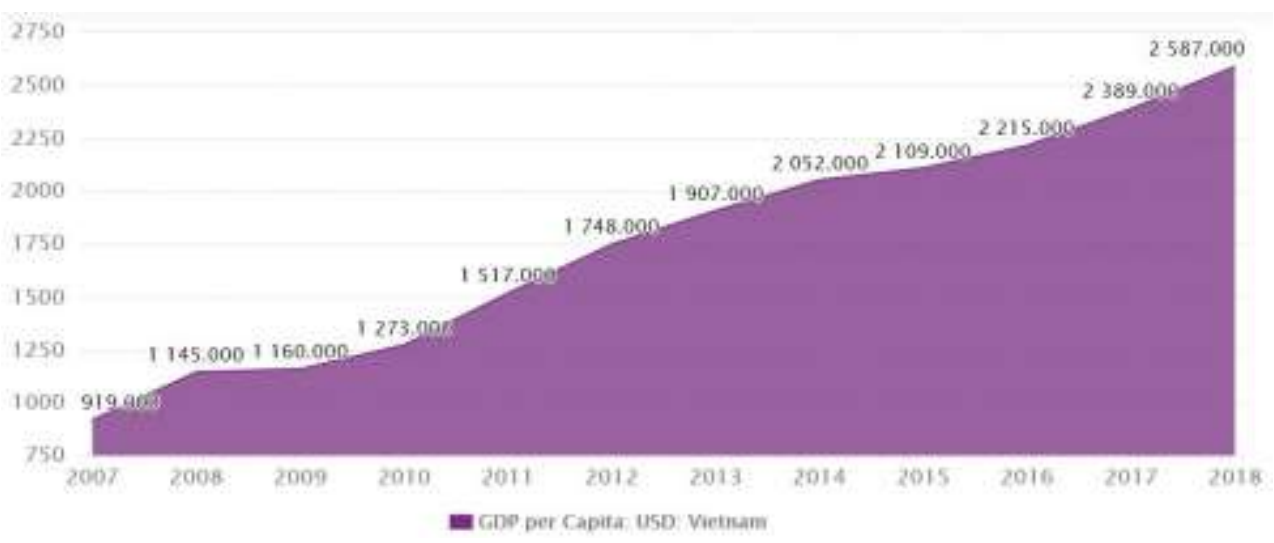

Gráfico 2: Crecimiento del PBI de Vietnam en millones de dólares (2007-2018) Fuente: www.ceicdata.com

\footnotetext{
${ }^{4}$ https://www.worldbank.org/en/news/press-release/2018/06/14/vietnams-economicprospect- improves-further-with-gdp-projected-to-expand-by-68-percent-in-2018 ${ }^{5}$ https://vietnamnews.vn/economy/518041/viet-nams-economic-growth-impressivein- q1.html\#3K8eXXM7iEgWFwwx.97
} 


\begin{tabular}{r|r|}
\hline \multicolumn{2}{|c|}{ Evolución PBI de Vietnam } \\
\hline Año & \multicolumn{1}{c|}{ Crecimiento $\%$} \\
\hline 2007 & 7,13 \\
\hline 2008 & 5,66 \\
\hline 2009 & 5,4 \\
\hline 2010 & 6,42 \\
\hline 2011 & 6,24 \\
\hline 2012 & 5,24 \\
\hline 2013 & 5,42 \\
\hline 2014 & 5,98 \\
\hline 2015 & 6,68 \\
\hline 2016 & 6,21 \\
\hline 2017 & 6,81 \\
\hline 2018 & 7,08 \\
\hline Promedio & 6,19 \\
\hline
\end{tabular}

Tabla 2: Crecimiento del PBI de Vietnam en porcentajes (2007-2018)

Fuente: elaboración propia con datos del Banco Mundial

Una característica que vale la pena destacar de la experiencia vietnamita es el énfasis en el incremento de la productividad que le han dado los actores políticos de ese país. En el Vietnam Development Forum, el primer ministro, Nguyen Xuan Phuc, enfatizó el rol de la productividad en la conformación de bases sólidas que permitan mejorar la competitividad del país. ${ }^{6}$ El Premier, además, enfatizó la importancia de llevar a cabo reformas financieras y bancarias para orientar los flujos de capital hacia industrias de alta productividad, así como proveer acceso al financiamiento a pequeñas y medianas empresas.

El sector manufacturero juega un rol destacado en la estructura económica de los países de la ASEAN. A pesar de una pequeña caída en el índice de compras de los gerentes (índice que mide especialmente el desempeño del sector manufacturero), que ralentizó o redujo levemente el crecimiento de esta variable en algunos de los países miembros, ${ }^{7}$ podemos ver en el siguiente gráfico que la ASEAN mantiene un promedio saludable de casi 50 puntos. Esto lo coloca actualmente por encima del promedio de la Zona euro (46,9), y otras economías emergentes, como Rusia $(45,6)$ y Sudáfrica $(48,6) .^{8}$

${ }^{6}$ https://www.vir.com.vn/in-record-2017-vietnam-living-up-to-its-potential55244.html

7 https://www.nationthailand.com/noname/30374026

${ }^{8}$ https://www.markiteconomics.com/public?language $=\mathrm{es}$ 


\section{LOLA IBÁÑEZ}

\begin{tabular}{|c|}
\hline Asia \\
America \\
Latina
\end{tabular}

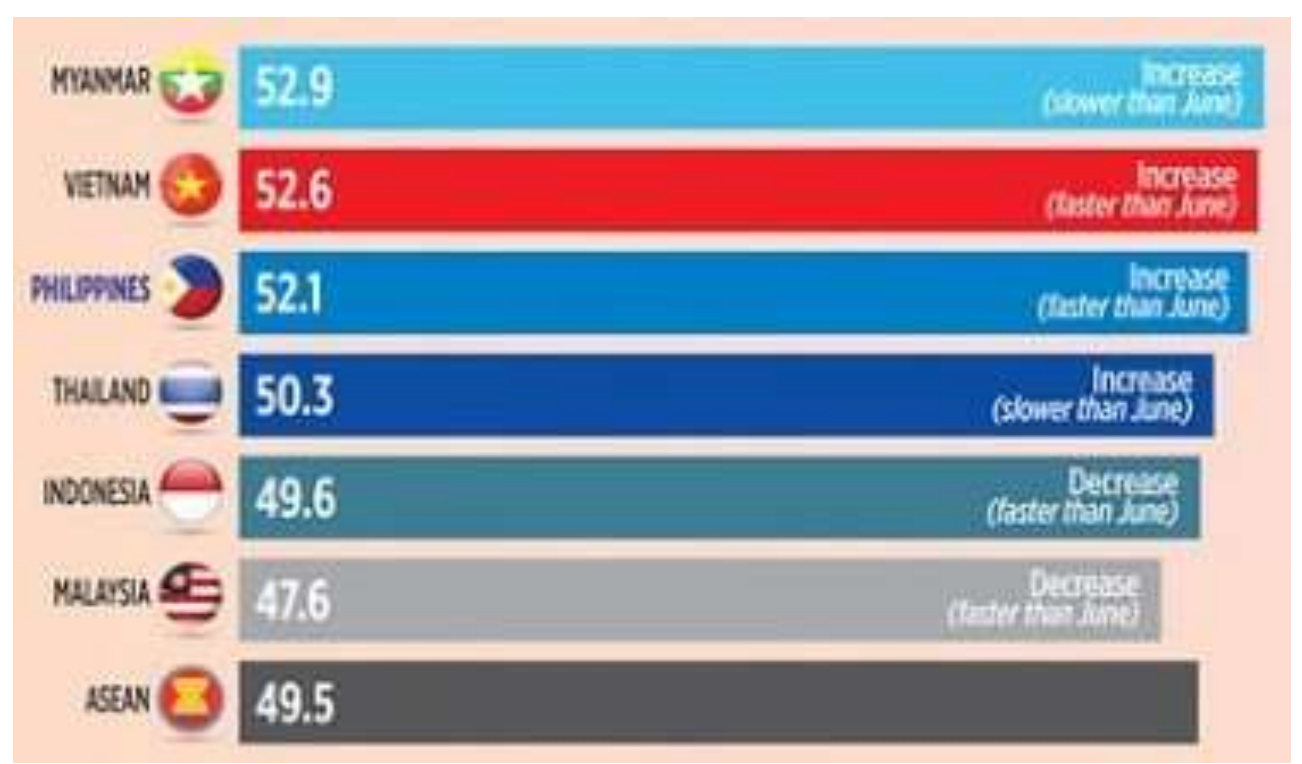

Gráfico 5: Purchasing Managers' Index por país y promedio regional Fuente: www.bworldonline.com

Si bien la ASEAN tiene un lugar estratégico en la cadena de valor global, el consumo doméstico y las inversiones jugaron un rol primordial para que el crecimiento en 2018 se mantuviera por encima del 5\%. ${ }^{9}$ En algunos países miembros como Filipinas el consumo doméstico contribuye a alrededor del $70 \%$ de la producción nacional, por lo que, muchas veces, estos incrementos en el PMI responden a incrementos de la demanda interna, que reflejan el aumento del poder de compra de los ciudadanos y medidas impositivas expansivas, como la reducción de impuestos sobre los ingresos personales, como los implementados por el gobierno filipino en enero de $2018 .^{10}$

Por otro lado, los flujos de inversión externa directa (IED) son otro aspecto de relevancia a tener en cuenta. En las últimas décadas, la ASEAN se ha transformado en un destino atractivo para los flujos de capitales. Solamente entre 2016 y 2017, el total de flujos de IED hacia la ASEAN se elevó de 123.000 millones de dólares a 137.000 millones; en paralelo, el flujo de IED desde la ASEAN hacia economías en desarrollo aumentó de 18\% en 2016 a $20 \%$ en 2017 , y los flujos hacia sur y este de Asia subieron del $31 \%$ al $34 \%$ en los mismos años. ${ }^{11}$

\footnotetext{
${ }^{9}$ https://asean.org/storage/2019/06/AEIB_5th_Issue_Released.pdf

10 https://www.bworldonline.com/phl-factory-gain-strongest-in-6-months /

${ }^{11}$ https://asean.org/storage/2018/11/ASEAN-Investment-Report-2018-for-
} 


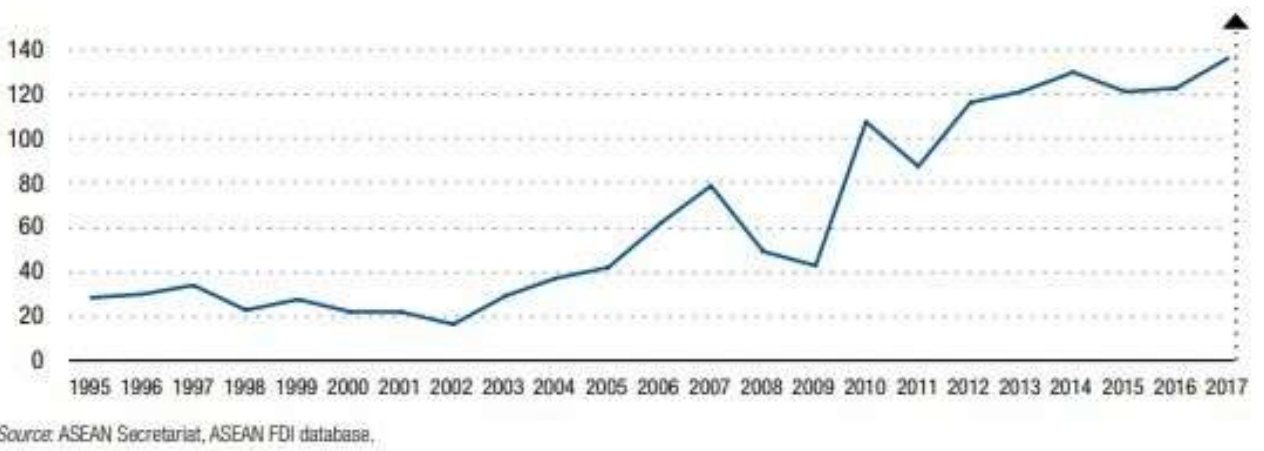

Gráfico 6: Evolución del volumen de inversión externa directa en la ASEAN Fuente: ASEAN Investment Report 2018

En 2017, solamente tres Estados (Singapur, Indonesia y Vietnam) daban cuenta del $72 \%$ de los flujos de IED hacia la ASEAN, lo cual muestra un alto nivel de concentración en su distribución. No obstante, esto representa una menor proporción en comparación con el 79\% que regía entre 2012 y 2016. Por su parte, Singapur se mantuvo como como el mayor recipiente de IED, concentrando el 45\% de los flujos destinados a la ASEAN incluso con la caída experimentada de 77.000 a 62.000 millones de dólares de inversiones.

Es sumamente interesante rastrear la evolución que han tenido no solo los indicadores macroeconómicos en los países de la ASEAN, sino también los indicadores sociales y de desarrollo. Si bien crecimiento económico no equivale a desarrollo, los países miembros han procurado un esquema de liberalización comercial con desarrollo de su estructura productiva, lo que les provee una mejor base desde la cual incrementar su productividad y competir a nivel global.

Con una mirada previsora, los países de la ASEAN supieron traducir el sostenido crecimiento económico del que goza esta región en un aumento del bienestar de la población, reduciendo el desempleo y aumentando el poder de compra, aumentando la proporción invertida del PBI en educación y reduciendo drásticamente (y en algunos caso prácticamente extinguiendo) el índice de analfabetismo, al mismo tiempo que incrementaron los índices de ingreso a la educación superior, ${ }^{12}$ esto en cuestión de pocas décadas. Asimismo, el cambio climático ${ }^{13}$ y el bienestar y la incorporación más activa de la juventud

\footnotetext{
Website.pdf

12 https://ched.gov.ph/wp-content/uploads/2017/09/2016-ASEAN-Indicators-asof-8-August-2017.pdf

13 https:/ / environment.asean.org/wp-content/uploads/2018/01/SOER5.pdf
} 
asiática, ${ }^{14}$ la infraestructura y la urbanización ${ }^{15}$ se han transformado también en temas de agenda que actualmente ocupan los foros regionales para incrementar el desarrollo social del Sudeste Asiático.

\section{Integración monetaria y financiera en la ASEAN}

Desde inicios del 2000, los países del este y sudeste de Asia se han inclinado por la cooperación regional en asuntos financieros y la integración monetaria. La introducción del euro y las repercusiones de la crisis asiática fueron importantes catalizadores de estas inclinaciones. Esto demostró que es posible implementar una divisa propia y dio origen a cuestionamientos en torno a la alta dependencia de estas economías con respecto al dólar (Dieter, 2008).

De esta manera, monetarismo regional y acuerdos de libre comercio han cobrado particular importancia en el continente asiático. El establecimiento de la Comunidad Económica de la ASEAN (AEC) en 2015, significó un hito mayor en términos de la constitución de un mercado regional de más de 622 millones de personas y equivalente a 2.6 billones de dólares, esto lo posicionó como el tercer mercado más grande de Asia y el séptimo nivel mundial. ${ }^{16}$ Más economías integradas significa un mercado más grande, lo que representa también más recursos disponibles para hacer frente a los problemas que se presenten.

Coincidimos con Heribert Dieter (2008) cuando afirma que la integración monetaria y financiera de la ASEAN se concentra en metas determinadas: 1) facilitamiento del comercio de bienes y servicios mediante la provisión de condiciones de estabilidad monetaria, 2) aseguramiento de mercados financieros eficientes y de buen funcionamiento, 3) prevención de crisis financieras y 4) dirección regional de crisis de crédito y divisas.

Así, el emergente monetarismo regional apunta a conservar la estabilidad financiera regional, incluso en un contexto de inestabilidad financiera global, prevenir ataques especulativos sobre las monedas nacionales y posibilitar a los países miembros un margen de maniobra con respecto al tipo de cambio, para formular políticas sostenibles y estables en un escenario de crisis y contagio (Dayaratna-Banda y Whalley, 2007).

Para amplificar el alcance de las capacidades y estrategias nacionales, los gobiernos tienden a crear coaliciones con otros gobiernos con los que poseen afinidades de distinta índole y así, aumentar su poder de negociación. Una región con peso en términos económicos, como lo es el Sudeste Asiático,

\footnotetext{
14 https://asean.org/storage/2017/10/ASEAN-UNFPA_report_web-final-05sep.pdf 15 https://asean.org/storage/2019/10/Investing_in_ASEAN_2019_2020.pdf

16 https://asean.org/asean-economic-community/
} 
representaría un potencial incremento en la influencia sobre la dirección de la gobernanza global. Esto, aunado a una coalición política coherente, otorgaría un considerable poder de negociación para posicionarse frente a las economías centrales (Kahler, 2010).

Más allá de las potencialidades de la ASEAN para influir en la gobernanza global, y más específicamente en la gobernanza financiera, primero es necesario que sus países miembros se planteen una estrategia coalicional viable con respecto a la potencia más cercana, China. Además, un desafío no menor se presenta al intentar alcanzar un consenso en relación con qué modelo de gobernanza promover como región, teniendo en cuenta la gran diversidad presente entre los países de la ASEAN.

Por lo pronto, los países del Sudeste parecen estar concentrando esfuerzos colectivos en la creación de mecanismos de cooperación financiera. Los primeros rastros de regionalismo financiero pueden rastrearse hacia 1977, cuando las autoridades monetarias de los cinco países originales de la ASEAN (Indonesia, Malasia, Filipinas, Singapur y Tailandia) implementaron acuerdos recíprocos de swaps, conocidos como ASEAN Swap Arrangements (ASA). ${ }^{17}$ Sin embargo, estos arreglos eran demasiado modestos como para amortiguar el impacto de la crisis financiera.

La Iniciativa Chiang Mai (CMI) fue creada en el año 2000, expandiendo el monto de los swaps bilaterales de ASA y extendiéndose a todos los nuevos miembros de la ASEAN, Corea del Sur, Japón y China (lo que comúnmente se conoce como la ASEAN+3). Además de una red de seguridad, la iniciativa fue pensada como una alternativa al Fondo Monetario, organismo que despertaba profundo recelo y reparos luego de la crisis de los noventa, las políticas de ajuste que había requerido como condición por sus préstamos y las consecuencias de éstas. Aunque frente a la crisis de 2008, economías como Corea del Sur y Singapur prefirieron recurrir a la Reserva Federal.

En 2009, la Iniciativa Chiang Mai se multilateralizó, transformándose en la CMIM (Chiang Mai Initiative Multilateralization). Un acuerdo de pooling de reservas donde los países de la ASEAN contribuirían con el $20 \%$ de los fondos, y los +3 con el 80\%, lo que en 2010 representaba 120.000 millones de dólares, accesibles a los países miembros como alternativa a otras fuentes de divisas estadounidenses. En 2011, se creó una unidad de vigilancia conocida como la Oficina de Investigación Macroeconómica de la ASEAN+3 (AMRO); y en 2012, se implementó el Encuentro de ministros de Hacienda y directores de los Bancos Centrales de la ASEAN +3, un foro común donde los responsables de la política económica y fiscal se reunían periódicamente.

En mayo de 2019, en una reunión del Encuentro de ministros decidió que la CMIM también contemplaría swaps en moneda local, no solo dólares

17 https://asiancenturyinstitute.com/economy/248-chiang-mai-initiative-an-asian-imf 
estadounidenses. ${ }^{18}$ Esta decisión refleja no solo la importancia progresiva del yuan en los intercambios regionales, sino también un producto de la incertidumbre global generada por la guerra comercial entre China y Estados Unidos. Esto responde a las metas regionales que Heribert Dieter (2008) detecta, especialmente aquellas referidas a la facilitación de los intercambios regionales y la conservación del buen funcionamiento y eficiencia del mercado financiero.

A pesar de los avances realizados en materia de integración monetaria para la estabilidad financiera, aún existen barreras que impiden que los mecanismos de Chiang Mai, aunque potencialmente beneficiosos, se desempeñen con efectividad. Un primer problema, lo presenta el nivel de los fondos disponibles a través de la CMIM, que continúan siendo demasiado modestos como para ser efectivos y resultar una alternativa plausible al FMI. ${ }^{19}$ Además, las marcadas diferencias en el tamaño de las economías al interior de la ASEAN pone en cuestión el tema del porcentaje de las reservas que deberá y podrá aportar cada miembro. Teniendo en cuenta que la protección de los socios más vulnerables y menos pudientes del esquema regional es esencial para la credibilidad y el funcionamiento de cualquier iniciativa.

Por otro lado, aunque la implementación de la Oficina de Investigación Macroeconómica sea una excelente herramienta para el monitoreo de las finanzas regionales, todavía carece de la fuerza necesaria para imponer sistemas de vigilancia y requerimientos a los actores que participan del circuito financiero. Si nos remitimos al acuerdo de creación de este órgano, podemos observar carece de la capacidad de acceder a cierta información empresarial que le resultaría vital en caso de grandes movimientos especulativos. ${ }^{20}$

Como podemos ver, la CMIM aún tiene camino que recorrer si quiere transformarse en una alternativa regional viable a los organismos de crédito de las economías centrales, pero no podemos dejar de reconocer los progresos que se han realizado en materia de integración monetaria para el mantenimiento de la estabilidad financiera. Asegurar un crecimiento sostenible y la consolidar los logros obtenidos son aspectos que los países en desarrollo no pueden darse el lujo de relegar en el orden de prioridades.

\section{Conclusiones}

No parece que las tendencias económicas favorables vayan a alterarse en un futuro cercano, aunque mantenimiento de la trayectoria de éxito económico y social dependerá (más allá del contexto global) de cómo los países

\footnotetext{
18 https://thediplomat.com/2019/05/reforming-the-chiang-mai-initiative/

${ }^{19}$ https://asiancenturyinstitute.com/economy/248-chiang-mai-initiative-an-asian-imf

${ }^{20}$ https://amro-asia.org/wp-content/uploads/2016/09/amro-agreement.pdf
} 
de la ASEAN enfrenten los desafíos que se plantean a mediano y corto plazo. Las perspectivas de crecimiento podrían verse afectadas si la región no puede alcanzar algún grado de coordinación política que le permita consolidar y expandir sus logros, y asegurar una prosperidad económica sostenible por medio del esquema regional.

Asia es una región sumamente heterogénea en todos los aspectos, comparada con Europa o América. La brecha entre países de renta baja y alta es mucho mayor que en cualquiera de las otras regiones a pesar de los avances obtenidos en las últimas décadas y, por ende, las diferencias de poder entre los países se hacen sentir constantemente.

Miles Kahler (2010) señala que otro desafío fundamental al que deberá hacer frente la ASEAN es la dependencia externa de la región, que constituye una característica estructural de su esquema económico. La organización de la cadena de valor global en la que se insertó la región continúa teniendo a las economías desarrolladas como destinos de exportación principales, lo cual genera una situación de fuerte dependencia con esas economías y vulnerabilidad ante las disrupciones que en ellas se produzcan. La apertura económica es una buena herramienta de desarrollo y una forma de proyectar presencia en el escenario internacional, pero esa apertura puede convertirse en una barrera al momento de construir un posicionamiento político coherente y cohesionado.

Además, la preferencia por un bajo grado de institucionalización al interior del esquema regional puede dificultar la consecución de un posicionamiento cohesionado. Las prácticas conocidas como la "manera ASEAN" de hacer las cosas (the ASEAN way) pueden resultar un arma de doble filo. Existe en la región un entendimiento en torno a los principios de no interferencia, que descansa en la creencia de que los problemas domésticos deben tener soluciones domésticas, ya que los Estados miembros son capaces de dar respuestas propias a sus propios problemas (Suzuki, 2019). Si bien este principio resulta razonable en algunos aspectos, las problemáticas que se generan en el ámbito financiero no responden a esta lógica. Como expusimos en la introducción, las características del sistema financiero actual son perfectamente capaces de trivializar los esfuerzos domésticos, si éstos no vienen acompañados de respuestas conjuntas.

En los últimos años, hemos presenciado la afluencia de fuerzas disruptivas del orden internacional establecido, lo cual no solo implica la emergencia y disputa del poder por parte de nuevas potencias, como China. Las tendencias proteccionistas y las oleadas nacionalistas que surgen en diferentes latitudes son un indicador preocupante de la preferencia por soluciones domésticas a problemáticas claramente globales.

Es de vital importancia promover soluciones conjuntas a cuestiones complejas, como aquellas relacionadas con el sistema financiero, y los procesos 
de integración regional establecidos conforman un espacio político propicio para alcanzar consensos básicos y dar visibilidad a discusiones fundamentales en el escenario internacional. Los países de la ASEAN pueden capitalizar sus logros socioeconómicos y aprovechar la coyuntura internacional para abogar por un nuevo paradigma de gobernanza, el cual sea capaz de proveer seguridad y estabilidad para que las economías en desarrollo se mantengan en la senda del crecimiento y el bienestar social.

\section{Referencias bibliográficas}

DIETER, H. (2014). ASEAN and the emerging monetary regionalism: a case of limited contribution. The Pacific Review. https:/ / doi.org/10.1080/09512740802294531

GARCÍA-ARIAS, J. (2008) Finanzas internacionales y desarrollo: ¿oportunidad o amenaza? Revista de la CEPAL https://repositorio.cepal.org//handle/11362/11285

GoldsteIN, M Y XIE, D. (2009). The impact of the financial crisis on emerging Asia. Peterson Institute for International Economics, 9-11.

https://www.piie.com/publications/wp/wp09-11.pdf

HenNing, R y KHAN, M (2011). Asia and Global Financial Governance.

Peterson Institute for International Economics, 11-16

https://www.piie.com/sites/default/files/publications/wp/wp1116.pdf

KAHLER, M (2010). Asia and the reform of Global Governance. Asian Economic Policy Review. https://doi.org/10.1111/j.1748-3131.2010.01160.x

León, C., Machado, C., Cepeda, F. y SARmiento, M. (2011). Too connected to fail Institutions and Payment System's stability: Assessing Challenges for Financial Authorities. Borradores de Economia (644) https:/ /dx.doi.org/10.2139/ssrn.2101221

Nesadurai, H (2008). The Association of Southeast Asian Nations (ASEAN). New Political Economy https://doi.org/10.1080/13563460802018588

O’Brien, R y Williams, M. (2004). Global Political Economy, evolution and dynamics. Macmillan.

STUBBS, R. (2014). The ASEAN alternative? Ideas, institutions and the challenge to global governance. The Pacific Review. https://doi.org/10.1080/09512740802294713

SUZUKI, S. (2019). Why is ASEAN not intrusive? Non-interference meets state strength. Journal of Contemporary East Asia Studies. https://doi.org/10.1080/24761028.2019.1681652 
Comité de ASEAN en Buenos Aires

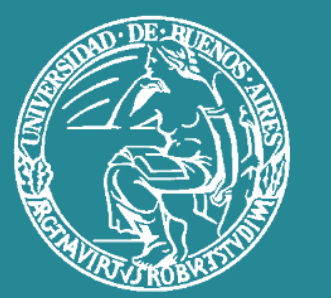

Grupo de Estudios sobre Asia y América Latina Instituto de Estudios de América Latina y el Caribe Universidad de Buenos Aires 